\title{
Lipomatosis intestinal: reporte de dos casos
}

\author{
Intestinal lipomatosis: Report of two cases
}

Lázaro Antonio Arango, MD, ${ }^{*}$ Claudia Patricia Díaz, MD, ${ }^{2}$ Mónica Londoño, MD, ${ }^{3}$ Pablo Ortiz, MD, ${ }^{4}$ Laura María Cano, MD. ${ }^{5}$

Gastroenterólogo clínico quirúrgico. Coordinador del Programa de Gastroenterología Clínico Quirúrgica de la Universidad de Caldas. Gastroenterólogo en la Unión de Cirujanos S.A.S Presidente ACED (2020-2022). Manizales, Colombia.

2 Gastroenteróloga clínico quirúrgica. Docente del Programa de Gastroenterología Clínico Quirúrgica de la Universidad de Caldas; Manizales, Colombia.

${ }^{3}$ Gastroenteróloga clínico quirúrgica. Universidad de Caldas; Manizales, Colombia.

${ }^{4}$ Residente de cirugía general, Universidad Militar Nueva Granada; Bogotá, Colombia. Rotante de gastroenterología en la Unión de Cirujanos S.A.S; Manizales, Colombia.

5 Médica concurrente de gastroenterología. Gastroenteróloga de la Unión de Cirujanos S.A.S; Manizales, Colombia.

*Correspondencia: Lázaro Antonio Arango, MD, lazaro.arango.m@gmail.com

Fecha recibido: $\quad 25 / 08 / 18$ Fecha aceptado: 31/05/19

\section{Resumen}

La lipomatosis intestinal es un término utilizado para describir una proliferación de tejido graso, histológicamente normal, en el tracto gastrointestinal. Se caracteriza por la presencia de tumores benignos, asintomáticos, de crecimiento lento y de origen subepitelial. En raras ocasiones, estos tumores pueden ser sintomáticos y asociarse a complicaciones importantes como obstrucción y sangrado gastrointestinal.

Así, la lipomatosis intestinal es una patología rara, con pocos casos reportados en la literatura y ninguno registrado en Colombia. A continuación, presentamos dos casos evaluados y seguidos en nuestro servicio de Unión de Cirujanos S.A.S de la Universidad de Caldas.

El primero es el caso de un hombre de 70 años con historia de episodios de dolor abdominal tipo cólico, asociados a distensión y diarrea posprandial. La endoscopia digestiva alta inicial reportó múltiples lesiones amarillentas, submucosas, en el duodeno, con diagnóstico histológico de lipomas. En este caso, se realizó una videocápsula endoscópica para determinar la distribución y las características de las lesiones presentes en todo el tracto gastrointestinal, así como para valorar la presencia de complicaciones.

El otro caso es el de un hombre de 81 años, quien ingresó a la institución por lipotimia y sangrado rectal. Por tanto, se realizó una endoscopia alta y una colonoscopia, cuyos resultados fueron normales. Luego de ello, se efectuó una videocápsula endoscópica que mostró lesiones lipomatosas; una de ellas, con sangrado y angiodisplasia al lado. Estas lesiones se manejaron mediante enteroscopia de doble balón y terapia con argón plasma.

\section{Palabras clave}

Lipomatosis, gastrointestinal, enfermedades gastrointestinales, endoscopia, enteroscopia de doble balón, colonoscopia.

\section{Abstract}

Intestinal lipomatosis is a proliferation of histologically normal fatty tissue in the gastrointestinal tract. It is characterized benign, asymptomatic, slow growing tumors with sub-epithelial origins. On rare occasions, they become symptomatic and can be associated with major complications such as gastrointestinal obstructions and bleeding. This rare pathology has been reported several times in the literature but not in Colombia prior to this study. We present two cases that were evaluated and followed up at the Unión de Cirujanos SAS and the University of Caldas in Manizales, Colombia.

The first is a 70-year-old man who had a history of colic associated with bloating and postprandial diarrhea. The initial upper digestive endoscopy reported multiple yellowish, submucosal lesions in the duodenum. His histological diagnosis was lipomas. In this case, an endoscopic video capsule determined the distribution and characteristics of the lesions throughout the gastrointestinal tract and assessed complications.

The second is an 81-year-old man who entered the institution due to lipothymia and rectal bleeding. Upper endoscopy and colonoscopy were normal, but a videocapsule endoscopy showed lipomatous lesions one of which was bleeding and had adjacent angiodysplasia. He was treated with double balloon enteroscopy and Argon plasma therapy.

\section{Keywords}

Lipomatosis, gastrointestinal, gastrointestinal diseases, endoscopy, double balloon enteroscopy, colonoscopy. 


\section{INTRODUCCIÓN}

El término lipomatosis intestinal se utiliza para describir una proliferación de tejido graso, histológicamente normal, localizada en cualquier parte del tracto gastrointestinal ( 1 , $2)$. Las lesiones se caracterizan por ser tumores benignos, de crecimiento lento y de origen subepitelial. La mayoría son asintomáticos y se diagnostican de manera incidental en estudios endoscópicos. Sin embargo, en ocasiones pueden presentar síntomas como dolor abdominal, distensión, constipación o diarrea, o manifestarse con complicaciones como sangrado gastrointestinal, intususcepción u obstrucción intestinal (1).

Estos tumores se localizan, principalmente, en el colon, el íleon y el yeyuno, mientras que si están en el duodeno se localizan en la segunda porción de este (3). La lipomatosis intestinal constituye una patología rara, con una incidencia en autopsias del 0,04-4,5\% (4) y con escasos reportes en la literatura médica; ninguno en Colombia.

Presentamos dos casos identificados en nuestro servicio y mostramos las imágenes y las características endoscópicas de la patología en mención, así como la utilidad de la enteroscopia de doble balón en el manejo del sangrado de uno de ellos.

\section{CASO 1}

Un hombre de 70 años, cuyo único antecedente patológico es cáncer de próstata, quien ha sido manejado quirúrgicamente sin complicaciones, y con una historia de varios años de dolor abdominal tipo cólico ocasional, asociado a episo- dios de distensión y diarrea posprandial. Además, ha presentado epigastralgia y pirosis y ha sido tratado con dieta y terapia farmacológica con trimebutina y un inhibidor de bomba de protones.

El paciente traía como estudio ambulatorio una tomografía axial computarizada (TAC) de abdomen contrastada, la cual fue reportada como normal, así como un coprológico seriado sin parásitos intestinales, y exámenes de química sanguínea generales normales.

Así pues, se ordenó realizar una endoscopia de vía digestiva alta (EVDA) y una colonoscopia, como parte del estudio del dolor abdominal. En la colonoscopia, solo identificamos hemorroides internas grado II, mientras que en la EVDA reportamos múltiples inclusiones amarillentas, submucosas, de, aproximadamente, $3 \mathrm{~mm}$ cada una (Figura 1), localizadas en el duodeno, hasta el ángulo de Treitz.

Además, se tomó una biopsia que reportó la existencia de células de tejido adiposo maduro. Por tanto, se decidió realizar una videocápsula endoscópica, a fin de determinar de forma más precisa la extensión de las lesiones. El estudio mostró múltiples lesiones amarillentas, de diferente tamaño, de aspecto benigno y con características similares a las reportadas en la EVDA, en todo el intestino delgado hasta la válvula ileocecal (Figura 2).

\section{CASO 2}

Varón de 81 años, natural de Anserma (Caldas), que ingresó al servicio de urgencias por causa de un episodio sincopal, acompañado de movimientos tónico-clónicos. Este fue un episodio único, con un período postictal corto y una recu-
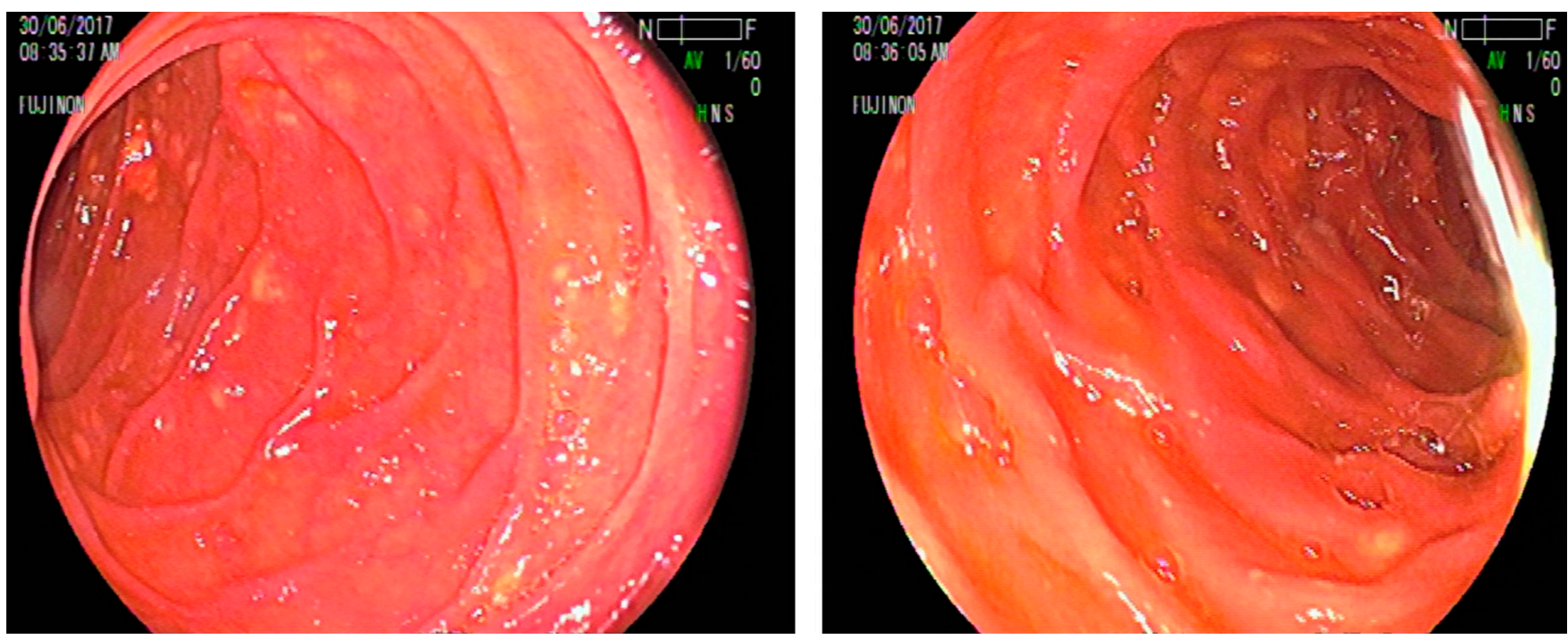

Figura 1. Imágenes de EVDEA que muestran múltiples inclusiones amarillentas, submucosas de aproximadamente 3 mm, localizadas en el duodeno. 

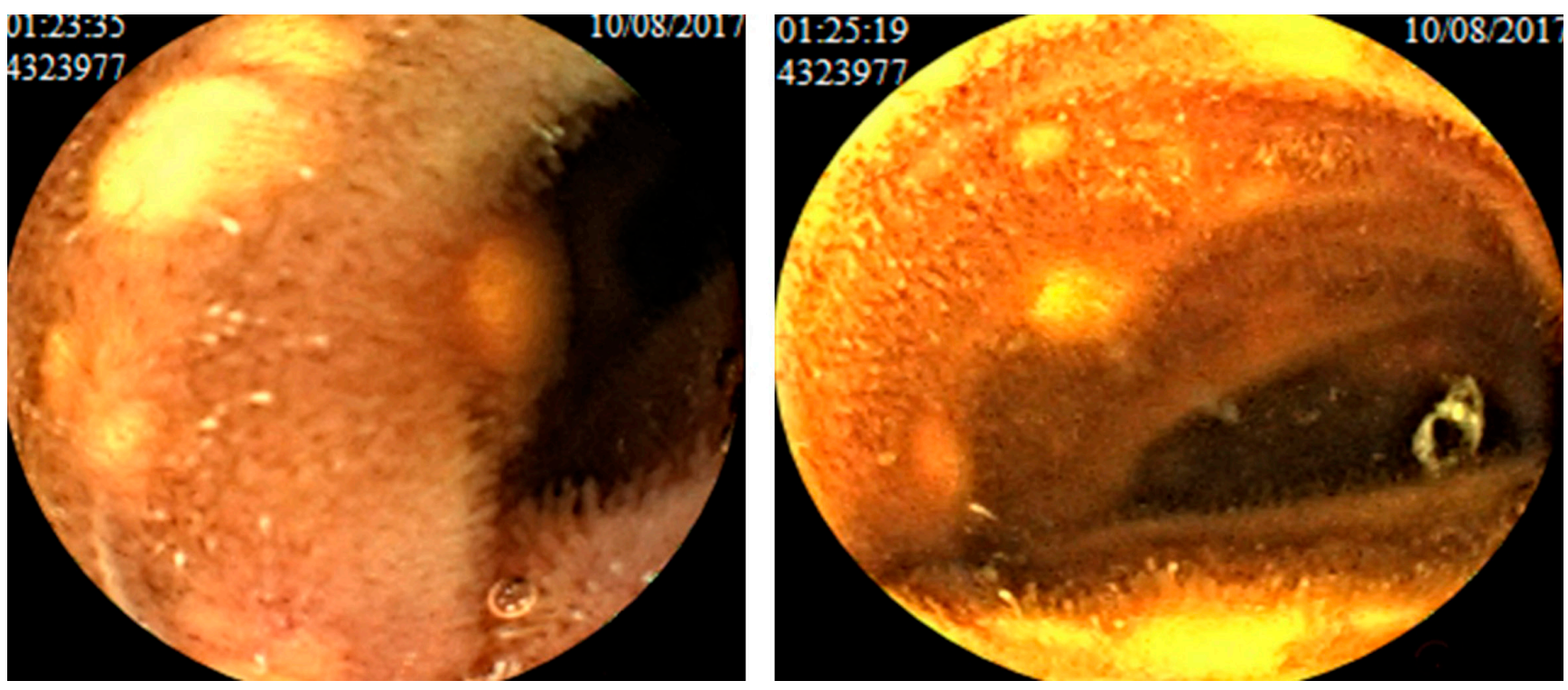

Figura 2. Imágenes de videocápsula endoscópica en las cuales se identifican múltiples lesiones amarillentas, redondeadas, de diferente tamaño y de aspecto benigno en el intestino delgado.

peración completa de la conciencia. Durante la revisión por sistemas, se informó la presencia de un sangrado rectal ocasional y escaso.

Asimismo, el paciente refirió como antecedentes la existencia de enfermedad coronaria de un vaso -con colocación de stent hace 9 años-, así como de enfermedad pulmonar obstructiva crónica (EPOC); Alzheimer; cáncer de próstata en manejo conservador; anemia crónica atribuida a pérdidas en el tracto gastrointestinal, causadas por una enfermedad diverticular y hemorroidal, y extabaquismo pesado durante 50 años.

El hombre negó que hubiese antecedentes familiares de patología gastrointestinal. Asimismo, aportó dos colonoscopias previas, en las cuales se informa una enfermedad diverticular del colon sigmoides y hemorroides internas de grado II.

En el momento del examen físico, el paciente presentó palidez cutánea, hipotensión, taquicardia y estigmas de sangrado fresco durante el tacto rectal. Además, se encontró un nivel de hemoglobina en 4,5 y hemoglobina corpuscular media de 27,7. La tomografía cerebral fue normal.

Durante la hospitalización, el paciente presentó múltiples episodios de hipotensión y dolor torácico, junto con 3 episodios de deposiciones melénicas abundantes, por lo que requirió la transfusión de 7 unidades de glóbulos rojos empaquetados. Se realizaron una EVDA y una colonoscopia total, en las cuales no se encontró la fuente del sangrado.

En consecuencia, se decidió realizar una videocápsula endoscópica, cuyo reporte describió la presencia de múltiples lesiones amarillentas, unas planas y otras elevadas, desde el yeyuno alto y 3 en el íleon. De igual forma, se encontró una lesión en el yeyuno medio como posible causa del sangrado, además de algunas angiodisplasias. Estos hallazgos fueron compatibles con una lipomatosis intestinal y malformaciones vasculares.

Se condujo al paciente a una enteroscopia de doble balón que mostró las lesiones descritas por la cápsula (Figura 3). Además, se realizó una terapia con argón plasma para el manejo de la lesión lipomatosa ulcerada y de una angiodisplasia. El paciente evolucionó satisfactoriamente y fue dado de alta.

\section{DISCUSIÓN}

El término lipomatosis intestinal fue descrito por primera vez por Hellstorm en 1906. Este hace referencia a una proliferación de tumores benignos, tipo lipomas, localizados en cualquier parte del tracto gastrointestinal (2). Los hallazgos endoscópicos incluyen múltiples masas submucosas redondeadas u ovaladas -que pueden ser sésiles o pedunculadas- y amarillentas, que protruyen a la luz intestinal (5). La mucosa que recubre generalmente a dichas masas es normal. Sin embargo, en ocasiones estas pueden ulcerarse.

En la TAC abdominal, pueden observarse como lesiones redondeadas $\mathrm{u}$ ovaladas bien definidas, con una densidad homogénea grasa ( -50 a -100 unidades Hounsfield). Este estudio se utiliza principalmente para valorar la presencia de complicaciones como obstrucción intestinal o intususcepción $(2,5)$. 

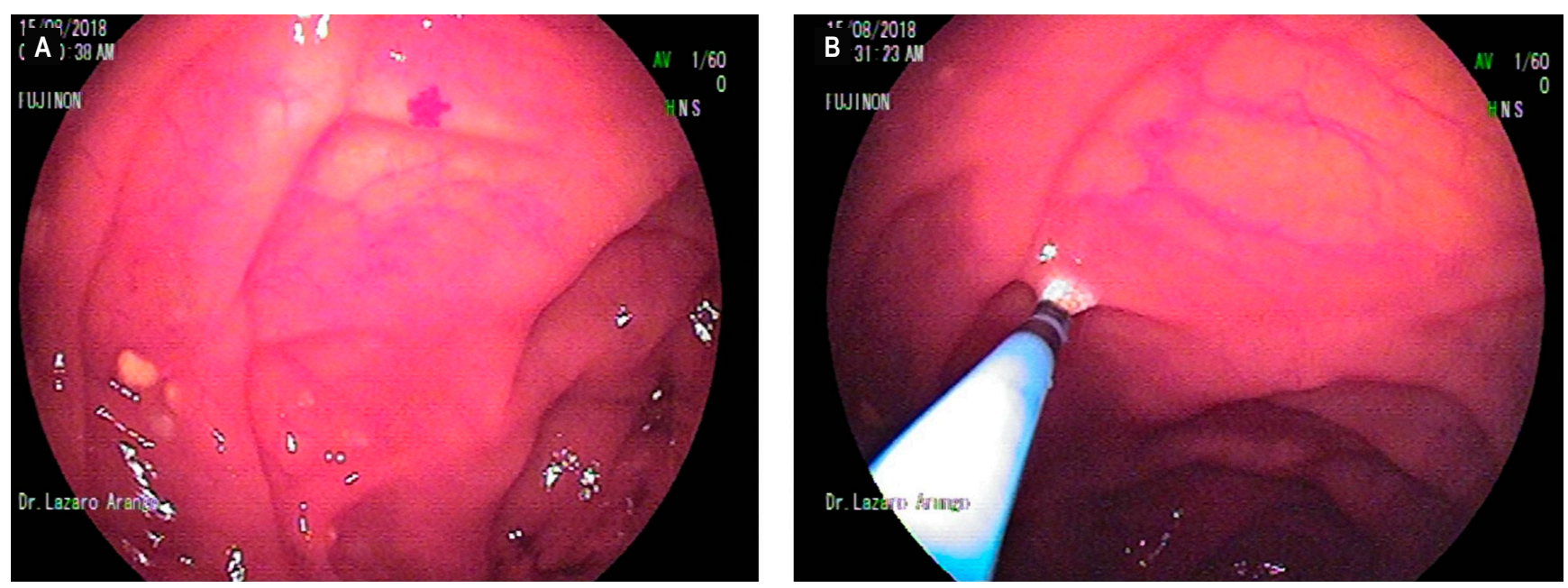

Figura 3. A. Lesiones lipomatosas del yeyuno y una angiodisplasia al lado. B. Terapia con argón plasma de la lesión lipomatosa ulcerada y de la angiodisplasia. Imágenes tomadas por el Dr. Lázaro Arango.

Durante el estudio histológico, se observa un tejido graso maduro normal, generalmente localizado en la submucosa (2). En el primer caso, el diagnóstico se realizó con la EVDA y la biopsia, pero se complementó con una videocápsula endoscópica. Entre tanto, en el segundo fue fundamental la videocápsula endoscópica para el diagnóstico y la enteroscopia de doble balón para el manejo del sangrado.

\section{CONCLUSIÓN}

La lipomatosis intestinal es una patología rara, generalmente benigna y asintomática, que en la mayoría de los casos solo requiere seguimiento clínico. Sin embargo, se puede presentar con complicaciones importantes como sangrado, intususcepción u obstrucción intestinal, que pueden requerir un manejo con procedimientos invasivos endoscópicos o quirúrgicos.
El diagnóstico se establece principalmente con la evaluación endoscópica y radiológica, que por tradición se ha realizado con EVDA, colonoscopia y tomografía abdominal. El presente artículo destaca la videocápsula endoscópica como un método eficaz y seguro para explorar todo el tracto gastrointestinal en pacientes con lipomatosis intestinal. Este método estaría indicado en pacientes sin complicaciones agudas, a fin de caracterizar las lesiones y determinar su extensión.

Asimismo, se enfatiza en la importancia de tener la posibilidad de realizar la enteroscopia de doble balón en los servicios que manejan pacientes urgentes y electivos gastrointestinales, a fin de complementar el diagnóstico $\mathrm{y}$ hacer un manejo adecuado de las complicaciones, tal y como ocurrió en el segundo caso descrito, en el cual se efectuó con éxito la terapia con argón de los sitios de posible sangrado.

\section{REFERENCIAS}

1. Zirpe D, Wani M, Tiwari P, Ramaswamy PK, Kumar RP. Duodenal Lipomatosis as a Curious Cause of Upper Gastrointestinal Bleed: A Report with Review of Literature. J Clin Diagn Res. 2016;10(5):PE01-PE4. http://doi.org/10.7860/JCDR/2016/19851.7881

2. Komagata T, Takebayashi S, Hirasawa K, Fukawa T, Arai M. Extensive lipomatosis of the small bowel and mesentery: CT and MRI findings. Radiat Med. 2007;25(9):480-483. http://doi.org/10.1007/s11604-007-0162-2

3. Krachman MS, Dave PB, Gumaste VV. Bleeding duodenal lipoma. J Clin Gastroenterol. 1992;15(2):180-181.
4. Kumar K, Noori MR, Patel KM, Yuen W, Bello C. Rare diagnosis of intestinal lipomatosis complicated by intussusception in an adult: A case report. Int J Surg Case Rep. 2017;39:339-342. http://doi.org/10.1016/j.ijscr.2017.08.038

5. Fang SH, Dong DJ, Chen FH, Jin M, Zhong BS. Small intestinal lipomas: diagnostic value of multi-slice $\mathrm{CT}$ enterography. World J Gastroenterol. 2010;16(21):2677-2681. http://doi.org/10.3748/wjg.v16.i21.2677 